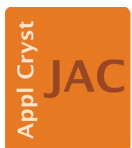

JOURNAL OF

APPLIED

CRYSTALLOGRAPHY

ISSN 1600-5767

Received 20 December 2018

Accepted 21 April 2019

Edited by A. Borbély, Ecole National Supérieure des Mines, Saint-Etienne, France

Keywords: three-dimensional X-ray diffraction (3DXRD); grain mapping; DCT; X-ray diffraction contrast microscopy; reconstruction schemes.

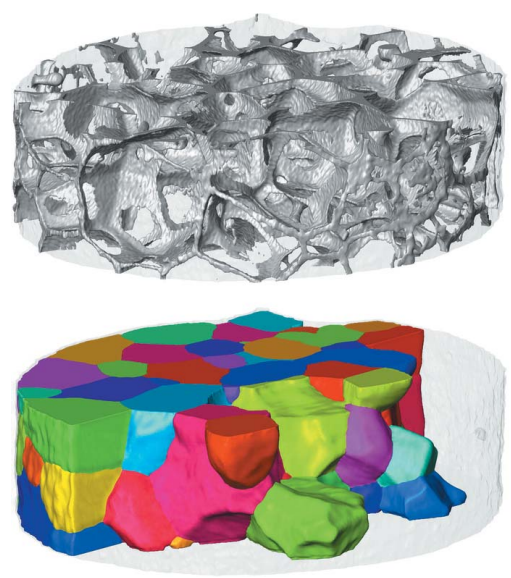

OPEN $\odot$ ACCESS

\section{D grain reconstruction from laboratory diffraction contrast tomography}

\author{
Florian Bachmann, ${ }^{\mathrm{a} *}$ Hrishikesh Bale, ${ }^{\mathrm{b}}$ Nicolas Gueninchault, ${ }^{\mathrm{a}}$ Christian Holzner ${ }^{\mathrm{a}, \mathrm{b}}$ \\ and Erik Mejdal Lauridsen ${ }^{\mathrm{a}}$
}

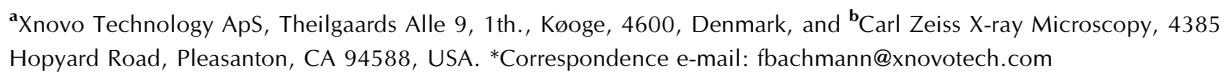

A method for reconstructing the three-dimensional grain structure from data collected with a recently introduced laboratory-based X-ray diffraction contrast tomography system is presented. Diffraction contrast patterns are recorded in Laue-focusing geometry. The diffraction geometry exposes shape information within recorded diffraction spots. In order to yield the three-dimensional crystallographic microstructure, diffraction spots are extracted and fed into a reconstruction scheme. The scheme successively traverses and refines solution space until a reasonable reconstruction is reached. This unique reconstruction approach produces results efficiently and fast for well suited samples.

\section{Introduction}

Over the past two decades, nondestructive volumetric orientation imaging techniques based on X-ray diffraction microscopy have evolved into well established tools for microstructure characterization of polycrystalline materials. Techniques originating at synchrotrons, able to spatially resolve phase, crystallographic orientation, stress and strain in the sample, have been demonstrated to be valuable for studying spatio-temporal relationships between crystallographic microstructure and material behaviour (Schmidt et al., 2004; King et al., 2008; Herbig et al., 2011). Utilizing a monochromatic high-energy parallel synchrotron beam, threedimensional X-ray diffraction (3DXRD) (Poulsen et al., 1997, 2001) and its variants high-energy diffraction microscopy (Suter et al., 2006; Bernier et al., 2011; Li \& Suter, 2013), scanning 3DXRD (Hayashi et al., 2015) and in particular X-ray diffraction contrast tomography (DCT) (Johnson et al., 2008; Ludwig, Reischig et al., 2009) have been shown to produce grain maps resolving grain-averaged orientation, shape and strain down to a minimum grain size of a few tens of micrometres. Lately, attempts to access intra-granular orientation (Li et al., 2012), also referred to as the full orientation field (Viganò et al., 2014, 2016), have been made. At the expense of probing volume, it has been shown that differential aperture X-ray microscopy (Larson et al., 2002; Ice et al., 2005) can resolve the orientation and strain field down to submicrometre level utilizing a polychromatic focused X-ray beam. Other approaches for grain mapping using a synchrotron source have been reported (Bleuet et al., 2008; Hofmann, Abbey et al., 2012; Hofmann, Song et al., 2012; Sanchez et al., 2014; Ferreira Sanchez et al., 2015).

The DCT technique (Ludwig et al., 2008; Johnson et al., 2008) has recently been adapted to laboratory scale. This 
adaptation, known as laboratory diffraction contrast tomography (LabDCT) (King et al., 2013; van Aarle et al., 2015), has been made commercially available as an additional imaging modality on an X-ray microscope (Holzner et al., 2016; McDonald et al., 2015). A polychromatic divergent cone beam emitted by a laboratory micro-focus X-ray source illuminates a millimetre-sized sample. Diffracting grains in the sample form astigmatically magnified spots on the detector (King et al., 2013). Whereas King et al. (2013) used a rather large magnification, where the source-to-rotation-axis and rotation-axis-to-detector ratio ranges were of the order of 1:51:25, McDonald et al. (2015) utilized the Laue-focusing geometry with a $1: 1$ ratio, resulting in diffraction patterns with elongated diffraction spots. Once grain centroid positions and crystallographic orientations have been found, the shapes of grains are typically reconstructed with algebraic reconstruction techniques (Poulsen \& Fu, 2003; Ludwig, King et al., 2009; King et al., 2010; Viganò et al., 2014) like the simultaneous iterative reconstruction technique (King et al., 2013; van Aarle et al., 2015), formulating a system of equations with a suitable forward projector and minimizing an objective function in some sense. Another approach involves maximizing a confidence function ( $\mathrm{Li} \&$ Suter, 2013), also known as a completeness function, which is the ratio between the observed and expected number of reciprocal vectors associated with a grain orientation (Poulsen et al., 2001; Schmidt, 2014).

Here, a versatile reconstruction scheme for grain mapping, named fast geometric indexing, is presented. A simplified diffraction model of a polycrystalline microstructure is considered, wherein acquired data are reduced to binarized diffraction contrast patterns. Spot intensities as well as interactions between grains are neglected and scattering contributions of individual grains are treated independently. The diffraction geometry becomes essential and is rigorously exploited in order to optimize the crystallographic orientation for each grain occupying space by maximizing its completeness. As a consequence, the reconstruction scheme systematically traverses sample space, successively indexing and mapping out grain by grain. Finally, the implementation of the reconstruction scheme is tailored and demonstrated specifically for LabDCT data. The reconstruction scheme extends the previously reported capabilities of extracting grain-based centroid and crystallographic orientation information from LabDCT data recorded in Laue-focusing geometry (McDonald et al., 2015); now, the morphology of the grains can also be resolved, rendering a full $3 \mathrm{D}$ crystallographic microstructure.

\section{Laboratory diffraction contrast tomography}

\subsection{X-ray diffraction imaging}

Contrary to conventional absorption contrast tomography, which deals with geodesic beam paths and gives rise to the Radon transform, diffraction contrast tomography addresses diffracted beam paths, where the signal is a superposition of the rather complex diffraction patterns of a crystalline sample.
The signal response of a detection surface area $P$, i.e. a detector pixel, is related to the integral intensity

$$
I(P)=\int_{\mathbf{p} \in P} \int_{\mathbf{x} \in V} I(\mathbf{x}, \mathbf{p}) \mathrm{d} V \mathrm{~d} P+I_{\mathrm{BG}}
$$

of the diffracted intensity contributions $I(\mathbf{x}, \mathbf{p})$ from location $\mathbf{x} \in V$ of an illuminated volume $V$ of a sample pointing to $\mathbf{p} \in P$, as illustrated in Fig. 1, and a non-diffracting background intensity $I_{\mathrm{BG}}$. Consider an incident wavevector $\mathbf{k}_{0}$ and a reflected one $\mathbf{k}$, originating from a source point $\mathbf{x}_{0}$, being diffracted at location $\mathbf{x}$ and being detected at $\mathbf{p}$, i.e.

$$
\mathbf{k}_{0}=\frac{1}{\lambda} \frac{\mathbf{x}-\mathbf{x}_{0}}{\left\|\mathbf{x}-\mathbf{x}_{0}\right\|} \quad \text { and } \quad \mathbf{k}=\frac{1}{\lambda} \frac{\mathbf{p}-\mathbf{x}}{\|\mathbf{p}-\mathbf{x}\|},
$$

with wavelength $\lambda$ or X-ray energy $E=h c / \lambda$. Diffraction occurs only if the scattering vector

$$
\mathbf{q}=\mathbf{k}-\mathbf{k}_{0}
$$

coincides with a reciprocal-space vector $\mathbf{g}_{h k l}$ of the crystal lattice at location $\mathbf{x}$ satisfying the necessary Laue diffraction condition

$$
\mathbf{g}_{h k l}=\mathbf{q} \text {. }
$$

With Bragg's law this holds for a wavelength

$$
\lambda=2\left\|\mathbf{g}_{h k l}\right\|^{-1} \sin \theta,
$$

where

$$
\sin \theta=\frac{\mathbf{q} \cdot \mathbf{k}}{\|\mathbf{q}\|\|\mathbf{k}\|} .
$$

The reciprocal-space vector

$$
\mathbf{g}_{h k l}=\Gamma_{\omega} \mathbf{V U B h}
$$

arises from a crystal lattice plane $\mathbf{h}=(h k l)^{\mathrm{T}}$ oriented in space accordingly. The reciprocal-space vector $\mathbf{B h}$ of a single crystal with reciprocal basis matrix $\mathbf{B}$ is first rotated by a crystallographic orientation $\mathbf{U} \in \mathrm{SO}(3) / \mathrm{S}$, and then possibly a stretch tensor $\mathbf{V}$ and finally a rigid-body rotation $\boldsymbol{\Gamma}_{\omega}$ of the sample are applied. The order of multiplication of the rotation and stretch tensors is exchangeable (Bernier et al., 2011) and depends on the point of view. Computation of $\mathbf{q}$ given $\mathbf{x}$ and $\mathbf{p}$ is usually referred to as back projection, whereas computation of $\mathbf{p}$ given $\mathbf{x}$ and $\mathbf{g}_{h k l}$ exploiting the reflection

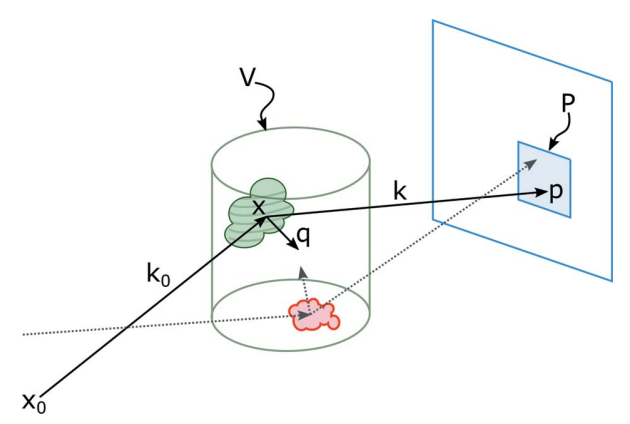

Figure 1

Sketch of diffraction geometry. Pixel $P$ counts the integral intensity of all grains in reflection condition contributing from all over the volume $V$. 


$$
\mathbf{k}=\mathbf{k}_{0}-2 \mathbf{g}_{h k l}\left(\mathbf{g}_{h k l} \cdot \mathbf{k}_{0}\right)
$$

is called forward projection (van Aarle et al., 2015). Notably, $\mathbf{g}_{h k l}$ and $-\mathbf{g}_{h k l}$ result in the same reflection.

Let the indicator function $1_{\mathbf{g}_{h k l}}(\mathbf{q})$ denote the condition that a crystal lattice plane $(h k l)$ diffracts:

$$
1_{\mathbf{g}_{h k l}}(\mathbf{q})= \begin{cases}1, & \text { if } \mathbf{g}_{h k l}=\mathbf{q}, \\ 0, & \text { otherwise, }\end{cases}
$$

where $\mathbf{q}$ is the back projection with regard to $\mathbf{x}$ and $\mathbf{p}$ computed from (3). Then, a simplified model for diffracted intensities $I(\mathbf{x}, \mathbf{p})$ from (1) can be formalized as the integral intensity,

$$
I(\mathbf{x}, \mathbf{p})=\int I_{0}(E) C(E) \sum_{h k l}\left[\left|F_{h k l}\right|^{2} 1_{\mathbf{g}_{h k l}}(\mathbf{q})\right] \mathrm{d} E,
$$

of a polychromatic beam with an X-ray energy intensity distribution $I_{0}(E)$. The sum goes over all crystal lattice planes $(h k l)_{i}, i=1, \ldots, N_{h k l}$, satisfying the diffraction condition (4), and $\left|F_{h k l}\right|^{2}$ is the scattering amplitude of the structure factor $F_{h k l}$. The intensity correction factor $C(E)=A(E) G(E) D(E)$ may include an energy-dependent attenuation intensity correction $A(E)$ along the diffracted beam path of the penetrated volume, polarization or geometric intensity corrections $G(E)$, and a detector intensity correction $D(E)$ related to the detection sensitivity and efficiency of the used hardware or other effects like point-spread response. Because of the rather selective nature of diffraction condition (4), integral (10) can be simplified to

$$
I(\mathbf{x}, \mathbf{p})=\sum_{h k l} I_{0}(E) C(E)\left|F_{h k l}\right|^{2} 1_{\mathbf{g}_{h k l}}(\mathbf{q}),
$$

where

$$
E=\left\|\mathbf{g}_{h k l}\right\| \frac{h c}{2} \frac{\|\mathbf{q}\|\|\mathbf{k}\|}{\mathbf{q} \cdot \mathbf{k}},
$$

with the entities described above.

\subsection{Grain mapping}

Traditionally, X-ray diffraction has primarily helped infer the lattice properties of a sample under investigation. With the aid of three-dimensional X-ray diffraction microscopy (Poulsen, 2004), it is possible to infer a spatially resolved model of the underlying crystallographic microstructure that describes phase, crystallographic orientation, stress and strain or further lattice properties of higher order (Poulsen, 2012). For grain mapping, the interest is to resolve the grain morphology and the crystallographic orientation in particular. The acquisition strategy seeks to record individual diffraction spots, which can be traced back to the grain of origin.

Several suggestions for experimental setups exist (Lauridsen et al., 2001; Larson et al., 2002; Suter et al., 2006; Johnson et al., 2008; Ludwig et al., 2008; King et al., 2013; Ferreira Sanchez et al., 2015; McDonald et al., 2015), which basically differ in the manner of beam formation in order to achieve individual diffraction spots and simplify the problem (1) with its model (10) further. Basic choices affecting the illuminated volume and diffraction geometry include a parallel pencil, line or box beam or a divergent fan or cone beam. The choice of $\mathrm{X}$-ray energy spectrum controls the range of reciprocal-space vectors to satisfy the Laue diffraction condition in the polychromatic case or the Bragg diffraction condition in the monochromatic case. Also, it matters whether the detector is positioned in back-reflection or transmission mode. The experimental setup is targeted to emphasize specific properties pertaining to the investigated crystalline microstructure.

Regardless of which constraints are imposed on the experimental setup in order to acquire data of the type described by integral (1), its reconstruction is notoriously illposed. The problems are the imposed Laue class due to Friedel's law (Friedel, 1913), the indistinguishable superposition of diffraction signal (Sørensen et al., 2012), and the loss of signal caused by erroneous or incomplete sampling. The experimental setup greatly influences the design and implementation of reconstruction algorithms to resolve the underlying crystallographic microstructure.

Quantifying the intensity of the diffracted signal is a complex step when considering a polychromatic X-ray beam. Whereas for monochromatic X-rays with energy $E$ the source intensity $I_{0}(E)$ and detection intensity correction $D(E)$ presumably remain constant, in the case of polychromatic $\mathrm{X}$-rays these intensity terms could be difficult to determine experimentally and might only be known insufficiently. Moreover, incorporating absorption along the beam path might become cumbersome, since the X-ray energies in (12) presumably have to be deduced from the diffraction condition, involving assumptions about $\mathbf{g}_{h k l}$. Furthermore, a significant amount of background intensity $I_{\mathrm{BG}}$ might arise from sample scattering or fluorescence. Hence, it is appealing to replace problem (1) defining the intensity of a pixel $I(P)$ by the binary problem

$$
B(P)= \begin{cases}1, & \text { if diffraction signal } \\ 0, & \text { otherwise }\end{cases}
$$

or

$$
B(P)=\bigvee_{\mathbf{p} \in P} \bigvee_{\mathbf{x} \in V} B(\mathbf{x}, \mathbf{p}) .
$$

Here the diffracted intensities $I(\mathbf{x}, \mathbf{p})$ are replaced by

$$
B(\mathbf{x}, \mathbf{p})=\bigvee_{h k l} 1(E) 1_{\mathbf{g}_{h k l}}(\mathbf{q}) .
$$

$1(E)$ is an indicator function for a valid X-ray energy range, with $1(E)=1$ if $E_{\min }<E<E_{\max }$ and zero otherwise. Binarization immediately implies that data should be acquired such that indistinguishable contributions of several grains, i.e. diffraction spot overlap, are mostly omitted.

Given a series of observed binarized diffraction images $B_{\omega}, \omega=1, \ldots, N_{\omega}$, obtained from an experiment, the completeness ratio

$$
c(\mathbf{x} ; \mathbf{U})=\frac{\text { No. of observed signals }}{\text { No. of forward-projected signals }}
$$

at location $\mathbf{x}$ given crystallographic orientation $\mathbf{U}$ quantifies how much signal can be explained by $\mathbf{U}$. More formally, 


$$
c(\mathbf{x} ; \mathbf{U})=\frac{\sum_{\omega} \sum_{h k l} B_{\omega}\left(\mathbf{x}, \mathbf{p}_{h k l}^{\mathrm{fwd}}(\mathbf{x} ; \mathbf{U})\right)}{\sum_{\omega} \sum_{h k l} 1_{\omega}\left(\mathbf{x}, \mathbf{p}_{h k l}^{\mathrm{fwd}}(\mathbf{x} ; \mathbf{U})\right)},
$$

where the forward projection $\mathbf{p}_{h k l}^{\mathrm{fwd}}$ at location $\mathbf{x}$ for a set of reciprocal space vectors $\mathbf{g}_{h k l}$ of crystal lattice planes $(h k l)_{i}$, $i=1, \ldots, N_{h k l}$, determined by $\mathbf{U}$ intersects the observed binarized diffraction image $B_{\omega}$ and the virtual image $1_{\omega}$. Then, the crystallographic orientation $\mathbf{U}_{k}$ is the optimal solution set of the optimization problem

$$
\mathbf{U}_{k}=\underset{\mathbf{U} \in \operatorname{SO}(3)}{\arg \max } c\left(\mathbf{x}_{k} ; \mathbf{U}\right)
$$

at location $\mathbf{x}_{k} \in V$, generally referred to as indexing. Maximizing (18) in the monochromatic case has been addressed elsewhere (Lauridsen et al., 2001; Ludwig, Reischig et al., 2009; Moscicki et al., 2009; Bernier et al., 2011; Sharma et al., 2012; Li \& Suter, 2013; Schmidt, 2014). The general case with polychromatic X-rays adds degrees of freedom between the spatial location in the sample and the corresponding diffraction spots as they pass through an X-ray energy range. Nevertheless, a back-projection approach is still applicable for both a divergent and a parallel beam, from which high-confidence lattice plane normals can be employed to index crystallographic orientations using a local optimization scheme.

Assuming a perfect crystal lattice over a grain, i.e. a constant crystallographic orientation, let

$$
S=\left\{\left(\mathbf{x}_{1}, \mathbf{U}_{1}\right), \ldots,\left(\mathbf{x}_{K}, \mathbf{U}_{K}\right)\right\}
$$

be a seed list of centroid positions $\mathbf{x}_{k} \in V$ and the associated crystallographic orientations $\mathbf{U}_{k}$ of grains $k=1, \ldots, K$ contained in the volume $V$. Then, the crystallographic microstructure to reconstruct can be represented as a partition

$$
\mathcal{P}=\bigcup_{\left(\mathbf{x}_{k}, \mathbf{U}_{k}\right) \in S} \mathcal{R}\left(\mathbf{x}_{k} ; \mathbf{U}_{k}\right)
$$

into regions

$$
\mathcal{R}\left(\mathbf{x}_{k} ; \mathbf{U}_{k}\right)=\left\{\mathbf{x} \in V \mid c\left(\mathbf{x} ; \mathbf{U}_{k}\right)>c\left(\mathbf{x} ; \mathbf{U}_{j}\right) \text { for all } k \neq j\right\}
$$

of highest completeness. The reconstruction problem is then to identify centroid positions and their associated crystallographic orientations.

\subsection{Fast geometric indexing}

Notably, neglecting intensity results in a purely geometric approach without the need for exhaustive intensity calculations concerning the diffracting volume. Moreover, individual contributions of the diffracting volume are treated independently of each other. In order to reconstruct (20), the illuminated volume can be traversed successively over candidate seeds, identifying grains one by one.

Since the seed list $S$ of centroid positions and crystallographic orientations is a priori unknown, an appropriate algorithm design accounts for systematic sampling of locations in the illuminated volume, achieved by a subdivision scheme. The heuristic approximation algorithm in the following is based on the strategy to successively choose seed locations suggested by the subdivision scheme and to identify adjacent grains and fill the space occupied by them using the input parameters minimum completeness $c_{\text {min }}$, trust completeness $c_{\text {trust }}$ and drop-off $\delta$. In order to avoid multiple consecutive draws of the same grain filling the space, the trust completeness $c_{\text {trust }}$ is intended to circumvent locations where a correct solution can be taken for granted. In contrast, the minimum completeness $c_{\min }$ is intended to reject solutions if the crystallographic orientation of the maximized completeness is uncertain and too ambiguous. The drop-off parameter $\delta$ sets a lower prediction bound to the completeness controlling the presumed size of the region occupied by the anticipated grains of the partition $\mathcal{P}$. The reconstruction scheme goes as follows: Algorithm (fast geometric indexing)

(0) Put $\mathcal{P}^{(0)}=\{\}, 0 \leq c_{\min } \leq c_{\text {trust }} \leq 1,0 \leq \delta \leq 1, i=0$. Choose a set of locations $\mathbf{x}_{k} \in V, k=1, \ldots, K$ from an arbitrary grid.

(1) Select an unvisited location $\mathbf{x}_{k} \in V$ in volume $V$ with the lowest completeness $c\left(\mathbf{x}_{k}\right)<c_{\text {trust }}$.

(2) Optimize

$$
\mathbf{U}_{k}=\underset{\mathbf{U} \in \operatorname{SO}(3)}{\arg \max } c\left(\mathbf{x}_{k} ; \mathbf{U}\right) .
$$

If completeness $c\left(\mathbf{x}_{k} ; \mathbf{U}_{k}\right)<c_{\min }$ then go to (1).

(3) Grow region

$$
\mathcal{R}_{\delta}\left(\mathbf{x}_{k} ; \mathbf{U}_{k}\right)=\left\{\mathbf{x} \in V \mid c\left(\mathbf{x} ; \mathbf{U}_{k}\right)>c\left(\mathbf{x}_{k} ; \mathbf{U}_{k}\right)(1-\delta)\right\}
$$

with a drop-off $\delta$ around $\mathbf{x}_{k}$.

(4) Compute the weighted centre of mass

$$
\mathbf{x}_{\mathrm{c}}=\frac{\int_{\mathbf{x} \in \mathcal{R}_{\delta}} \mathbf{x} c\left(\mathbf{x} ; \mathbf{U}_{k}\right) \mathrm{d} V}{\int_{\mathbf{x} \in \mathcal{R}_{\delta}} c\left(\mathbf{x} ; \mathbf{U}_{k}\right) \mathrm{d} V}
$$

of the grown region $\mathcal{R}_{\delta}\left(\mathbf{x}_{k} ; \mathbf{U}_{k}\right)$.

(5) If $\left\|\mathbf{x}_{k}-\mathbf{x}_{\mathrm{c}}\right\|>\epsilon$ for a distance tolerance $\epsilon$ then go to (2) with replacement $\mathbf{x}_{k}=\mathbf{x}_{\mathrm{c}}$, else update partition

$$
\mathcal{P}^{(i+1)}=\mathcal{P}^{(i)} \cup \mathcal{R}_{\delta}\left(\mathbf{x}_{k} ; \mathbf{U}_{k}\right),
$$

increment $i=i+1$ and go to (1).

Depending on the selected location $\mathbf{x}_{k}$, for instance if $\mathbf{x}_{k}$ was randomly chosen close to a grain boundary, optimizing $\mathbf{U}_{k}$ in step (2) might result in an ambiguous solution set of possible candidates of crystallographic orientations. Growing a region around the $n$ topmost distinct candidate solutions in step (3) might actually deliver several regions at the same iteration step. Computation of the weighted centre of mass in step (4) moves the initial location iteratively to the optimal one. Note that the resulting partition $\mathcal{P}^{(i)}$ of this heuristic approach depends to some degree on the order of traversal.

The resulting partition $\mathcal{P}^{(i)}$ tends to be over partitioned, particularly in regions that are difficult to index. In such regions, optimization of $c\left(\mathbf{x}_{k} ; \mathbf{U}_{k}\right)$ is repeated multiple times because the trust completeness $c_{\text {trust }}$ cannot be overcome owing to missing information. Merging adjacent regions based on the misorientation angle of their crystallographic orientation (Bachmann et al., 2011) is a recommended post-processing procedure to identify the actual grains. Because of the model-based assumption of a constant crystallographic orientation within a region, this step should also be accompanied by a re-computation of the weighted mean orientation 
(Bachmann et al., 2010). Consequently, the completeness $c\left(\mathbf{x} ; \mathbf{U}_{k}\right)$ within a newly obtained merged region must also be updated in order to correspond to the diffraction spots within the binarized diffraction contrast patterns.

\subsection{Data acquisition}

The grain-mapping technique has been implemented in the laboratory on a ZEISS Xradia 520 Versa X-ray microscope (McDonald et al., 2015). The X-ray microscope is equipped with an additional specialized LabDCT imaging modality optimized to record diffraction contrast patterns. Data acquisition is usually performed in two steps. First, conventional absorption contrast tomography is performed in order to determine the illuminated sample volume $V$. Subsequently, diffraction contrast tomography with the identical acquisition geometry is performed with regard to the same illuminated sample volume $V$ but recording diffraction contrast patterns.

The X-ray source of the instrument is a transmission-type micro-focus tube with a tungsten anode, producing a polychromatic white cone beam. Though present, the characteristic emission lines are negligible features of the Bremsstrahlung spectrum, ranging from 10 to $160 \mathrm{keV}$. The sample is placed on a micro-positioning rotation stage between the source and the detector. A scintillator optically coupled to a high-sensitivity CCD camera with an effective pixel size of roughly $3.4 \mu \mathrm{m}$ detects X-rays transmitted, scattered and diffracted by the sample. The setup of the LabDCT imaging modality is sketched in Fig. 2. In order to enhance the contrast of the diffracted signal, an aperture mounted in front of the X-ray source defines a cone beam such that for the most part only the sample is illuminated. A centred beam stop shields the high-sensitivity scintillator from overexposure by the direct X-ray beam, and the diffracted signal is recorded in the remaining part. Usually 181 or more diffraction contrast patterns are acquired by rotating the sample once around in $2^{\circ}$ or finer steps. Typical working distances range from 12 to $25 \mathrm{~mm}$ equidistant from source to rotation axis and rotation axis to detector. In terms of absorption contrast tomography, this symmetric 1:1 distance ratio corresponds to a geometric magnification of factor 2. Uniquely associated with the divergent polychromatic cone beam, aligned lattice domains in the sample that form a grain and that satisfy the Laue diffraction condition act like lenses that bundle and converge the diffracted X-ray beam to a focal zone (Guinier \&

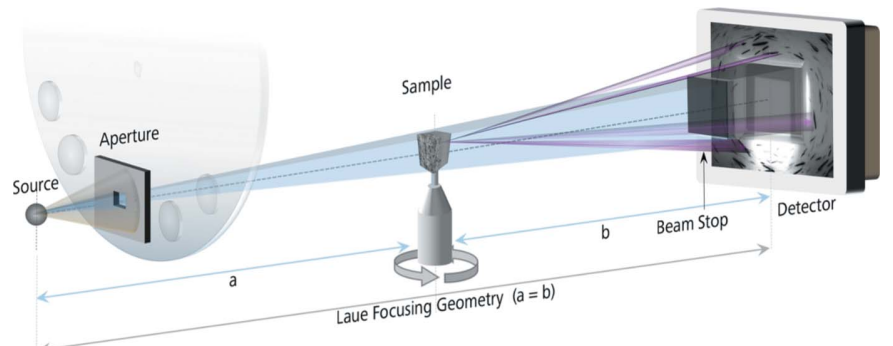

Figure 2

Setup of the LabDCT imaging modality.
Tennevin, 1949) in which the detector is placed. Typical diffraction contrast patterns recorded with this Laue-focusing geometry form elongated diffraction spots on the detector. The advantage of exploiting this Laue-focusing effect is that a higher number of Laue spots can be recorded with less overlap and a higher signal-to-noise ratio on the same projection. This is because a spot covers less space with higher integral intensity, and consequently a larger volume with shorter exposure times can be illuminated.

To accommodate imaging of a variety of different samples, a set of different-sized apertures and beam stops provides further means to adjust the illuminated volume and pattern quality in order to control the information content of diffraction contrast patterns formed on the detector.

\subsection{Implementation}

Acquired experimental data need to be prepared for the reconstruction algorithm. Extraction of the scattering volume from the reconstructed absorption contrast volume can be performed with standard segmentation methods. The extraction of binarized diffraction contrast patterns from raw projection images consists of several steps. These include a background correction, by subtracting a background estimated from a running median through the image stack (Johnson et al., 2008), exclusion of the beam stop area and finally a binarization of the pre-corrected diffraction contrast patterns, e.g. using a Laplacian of Gaussian segmentation approach (Lind, 2013). The latter is a crucial step and requires the operator's best judgement, such that the binarization does not compromise the shape profile of the spots, as it ultimately influences the completeness and thus the quality of the reconstruction.

The crystallography of the sample should be known in advance in order to specify the reciprocal-space vectors required for indexing. In particular, for non-cubic systems, unit-cell lengths and angles affect indexing significantly. As a rule of thumb, more than 15 reciprocal-space vectors are required for the setup described above to yield a reliable grain map that would also allow any possible pseudo-twin orientations to be identified (Schmidt, 2014). These vectors correspond to the three (body-centred cubic) or four (face-centred cubic, hexagonal close packed) strongest $\{h k l\}$ lattice plane families in the case of cubic or hexagonal symmetry.

The fast geometric indexing reconstruction algorithm described above is discretized on a voxelated volume of arbitrary choice. The set of locations to be traversed systematically is realized by means of a top-down hierarchical cubic close-packing subdivision scheme put onto the voxelated volume. The subdivision results in a set of levels, where the set of locations for each level $L$ is such that the Voronoi cell volume associated with the sampling locations is proportional to $2 \times 8^{L}$ voxels. Locations of the top level are traversed first, and the reconstruction is successively refined level by level. The procedures are implemented in the workflow-based commercially available software package GrainMapper $3 D$ (https://xnovotech.com/3d-crystallographic-imaging-software/). 

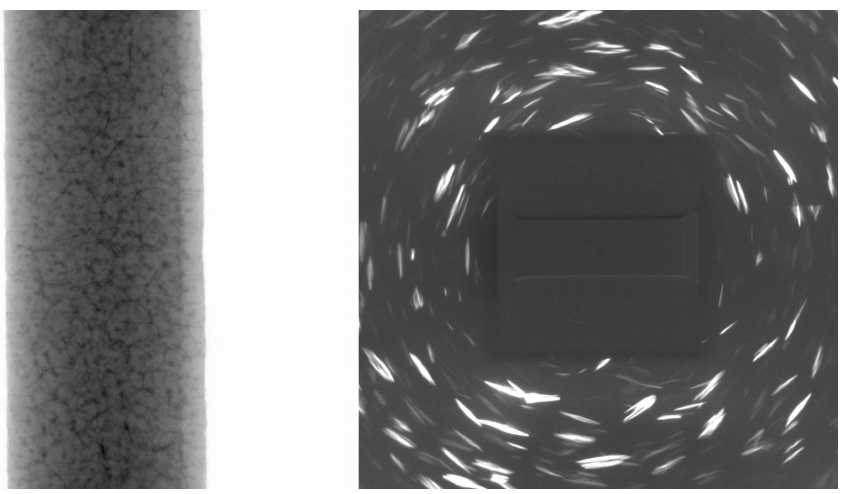

Figure 3

(Left) Acquired absorption projection and (right) diffraction contrast pattern.

\section{Results}

Collection of data was performed with a ZEISS Xradia 520 Versa X-ray microscope equipped with a LabDCT imaging module. An aluminium sample with grain boundaries decorated with copper ( $c f$. Dake et al., 2016) was chosen as a model material system to demonstrate the capabilities of LabDCT using the algorithm described. The sample was shaped into the form of a cylindrical rod with a $1400 \mu \mathrm{m}$ gauge diameter. The cylindrical sample was mounted on a standard pin vise, placed on the sample rotation stage, and positioned $14 \mathrm{~mm}$ away from both source and detector. An absorption scan was acquired with 1601 projections of $1 \mathrm{~s}$ exposure time each, within $1.5 \mathrm{~h}$. A LabDCT scan was acquired with 181 projections collected in $2^{\circ}$ steps with $300 \mathrm{~s}$ exposure time, lasting $15 \mathrm{~h} 40 \mathrm{~min}$. Both scans were performed at $160 \mathrm{kV}$ accelerating voltage and $62 \mu \mathrm{A}$ current. For the LabDCT scan, a $250 \times 750 \mu \mathrm{m}$-sized aperture was placed approximately $8.75 \mathrm{~mm}$ in front of the polychromatic point source, illuminating a height of approximately $380 \mu \mathrm{m}$ and a width of $1100 \mu \mathrm{m}$ on the axis of rotation of the sample. The DCT scan volume consisted of an illuminated volume of $0.573 \mathrm{~mm}$ of the aluminium rod. Parts of the sample located outside of the aperture field of view were effectively illuminated during a $360^{\circ}$ rotation in at least $65 \%$ of all DCT projections acquired. A $2.5 \times 2.5 \mathrm{~mm}$ beam stop was used to block the direct beam, as shown in the schematic of Fig. 2. Example projections taken in both the absorption and the diffraction contrast mode are displayed in Fig. 3. The effective aperture footprint on the detector is $0.8 \times 2.4 \mathrm{~mm}$ and the footprint of the beam stop approximately $3.0 \times$ $3.0 \mathrm{~mm}$.

The LabDCT reconstructions were performed with the Xnovo GrainMapper3D software package on a workstation equipped with a dual Intel Xeon E5-2650 v3 processor (40 threads) and 128 GB RAM. Diffraction contrast patterns were binarized with an adapted Laplacian of Gaussian spotextraction method (Lind, 2013). About 76500 spots were extracted from the 181 projections, i.e. approximately 420 per
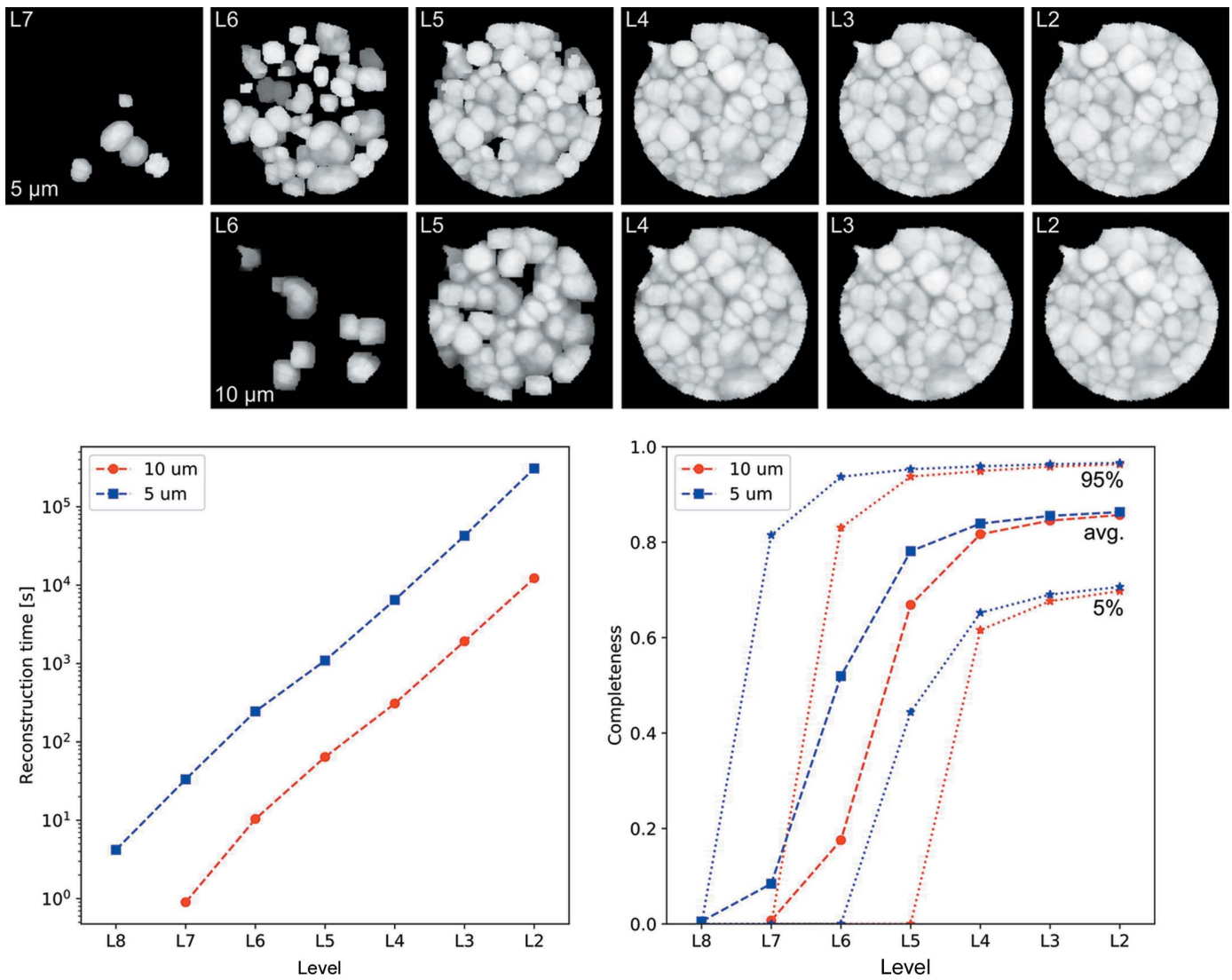

Figure 4

(Top) Completeness maps with 5 and $10 \mu \mathrm{m}$ resolution through reconstruction levels L7 to L2 and their corresponding (bottom left) reconstruction time and (bottom right) average completeness, 5 and $95 \%$ completeness percentiles. 
projection on average. A unit-cell length of $4.0496 \AA$ was used as input for the face-centred cubic aluminium (Wycko, 1963). The four strongest lattice planes, $\{111\},\{200\},\{220\}$ and $\{311\}$, were selected for indexing with 4,3,6 and 12 reciprocal-space vectors, respectively (half of their multiplicity), summing to 25 in total. Illumination calculations were performed in order to correct the completeness in a partially illuminated sample volume. The initial reconstruction was based on the calibration carried over from the instrument, and then the calibration was improved via self-consistent fitting, minimizing the residuals between observed and forward simulated diffraction spots.

The reconstruction scheme was tested for two scenarios, where the acquired data volume $V$ was subdivided into $160 \times$ $160 \times 37(947200)$ and $320 \times 320 \times 73(6773760)$ voxels with voxel edge lengths of 10 and $5 \mu \mathrm{m}$, respectively. Fig. 4 summarizes the reconstruction performance of each level $L$ using the reconstruction parameters trust completeness $c_{\text {trust }}=$ $85 \%$, minimum completeness $c_{\min }=45 \%$ and drop-off $\delta=2 \%$. The reconstruction algorithm starts with coarse sampling locations selected from a top level, such as L7, and proceeds by successively choosing refined sampling locations of the grid up to L2. For the aluminium-copper sample considered here, level L2 of the hierarchical subdivision scheme was completed after $3.5 \mathrm{~h}$ and $3 \mathrm{~d} 14 \mathrm{~h}$ for the 10 and $5 \mu \mathrm{m}$ reconstructions, respectively. Roughly 40000 and 280000 locations were selected and indexed in total. On average $\sim 3.4$ iterations per second of the reconstruction algorithm could be performed for $10 \mu \mathrm{m}$ voxel edge length and $\sim 0.9$ iterations per second for $5 \mu \mathrm{m}$ voxel edge length, which is a factor of $\sim 3.5$ slower compared to roughly $8 \times$ more voxels. The completeness maps in Fig. 4 show a gradual filling up of the volume, with reconstructed grains going hierarchically down from level to level as reconstruction progresses. After $2-2.5 \%$ of the total indexing attempts performed, i.e. approximately 1000 indexing attempts, an orientation and its corresponding completeness were assigned to more than $99.5 \%$ of all voxels in the reconstruction volume, which was the case after completing L4 for both reconstructions after $5 \mathrm{~min} 12 \mathrm{~s}$ and $1 \mathrm{~h} 50 \mathrm{~min}$, respectively. Less than $5 \%$ of all voxels have a completeness less than $60 \%$ after reaching $\mathrm{L} 4$, and more than half have a completeness higher than $80 \%$. Most information on the reconstructed crystallographic microstructure is gained after completing indexing of reconstruction level L4. The gain of information from reconstruction level L2 is negligible in proportion to the
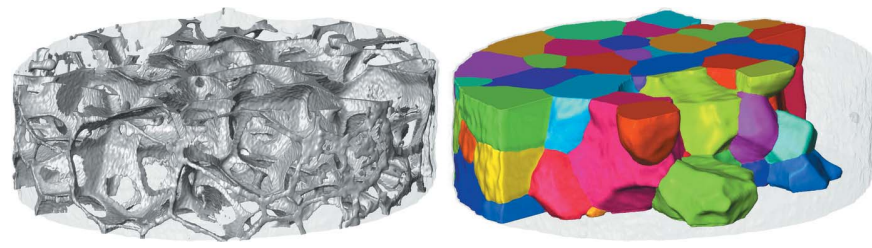

Figure 5

(Left) Absorption reconstruction and (right) DCT reconstruction of the $\mathrm{AlCu}$ sample in random colour coding. The illuminated cylindrical volume has a diameter of approximately $1400 \mu \mathrm{m}$ and a height of $400 \mu \mathrm{m}$ (volume $0.573 \mathrm{~mm}$ ). increase in computational time compared with level L3, completed after $32 \mathrm{~min}$ and $11 \mathrm{~h} 50 \mathrm{~min}$ for the 10 and $5 \mu \mathrm{m}$ reconstructions, respectively. Only a handful of additional small grains were identified at the expense of a 6-7 times longer computation time.

Fig. 5 illustrates the copper network along grain boundaries extracted from the reconstruction of absorption contrast data and the polycrystalline microstructure reconstructed from LabDCT data of 129 reconstructed grains from the aluminium-copper sample. The equivalent spherical diameter of the grains is $168 \mu \mathrm{m}$ on average, including partially illuminated grains at the top and bottom of the sample. The high contrast of the copper phase in the absorption contrast data was used to validate the grain-boundary location assessed from the LabDCT reconstruction. The overlay of the absorption contrast data on top of the LabDCT reconstruction in Fig. 6 shows a good agreement along grain boundaries. The accuracy of grain boundary location of the LabDCT reconstruction versus the absorption reconstruction is quantified in Fig. 7, based on the method proposed by Ludwig, Reischig et al. (2009). The average distance in the grain-boundary location between the two reconstructions was found be $7.6 \mu \mathrm{m}(1.52$ voxels), whereas $90 \%$ fall within a distance of $20 \mu \mathrm{m}$ (4 voxels).
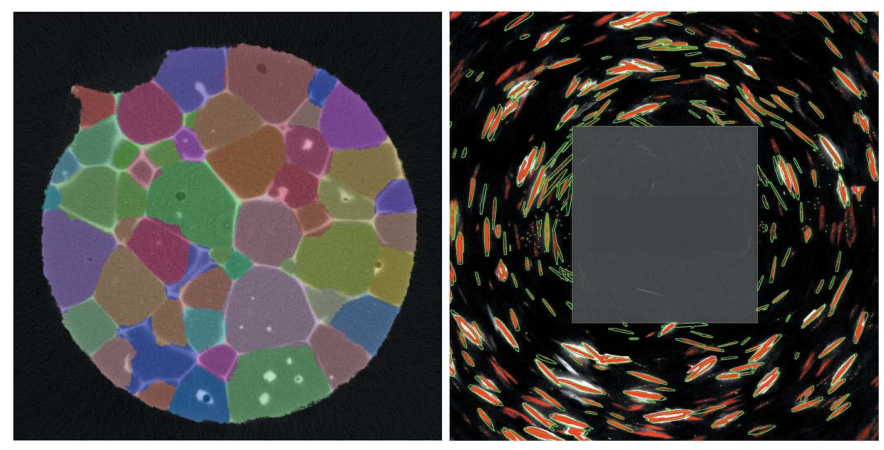

Figure 6

(Left) Overlay of absorption and colour-coded LabDCT reconstruction and (right) binarized diffraction images (red) and forward-projection outline (green) of the diffraction spot.

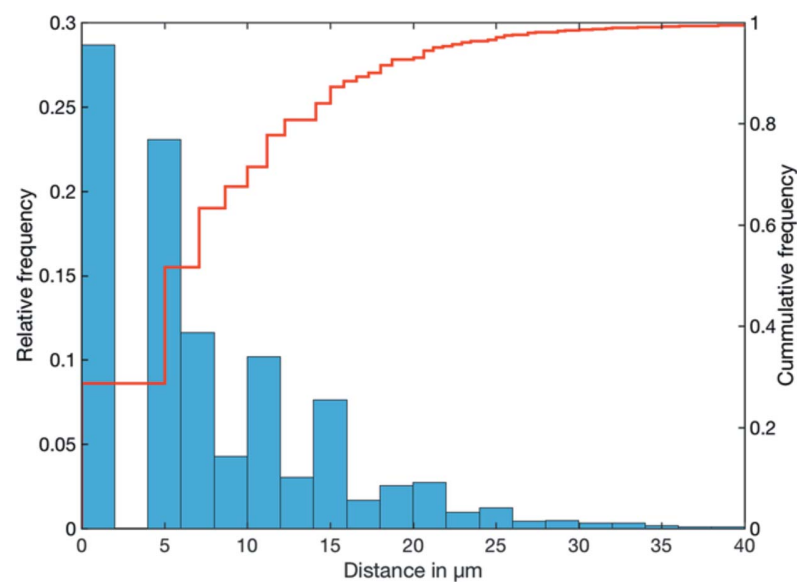

Figure 7

Histogram of the deviation in the grain boundary locations between the absorption and DCT reconstruction. 
The forward-projected outline of the first four lattice planes used for indexing of grain boundaries of the reconstructed polycrystalline microstructure in Fig. 6 explains most of the binarized diffraction spots and reveals a good agreement in position, size and shape. For the whole LabDCT data set, between 155 and 240 (90\%) binarized diffractions spots, and 198 on average, were attributed to individual grains. Some of the weaker diffraction spots close to high-intensity spots are not properly binarized, as the adapted Laplacian of Gaussian spot-extraction method did not separate them into individual spots. Unexplained diffraction spots arise either from higher lattice planes or potentially from small grains missed by the indexing, because the signal for higher-order lattice planes is incomplete as a result of their smaller scattering volume.

Some of the high-angle grain boundaries are observed to be only decorated partially with copper, whereas low-angle boundaries are observed generally to be not decorated and could only be revealed by LabDCT. Grain clusters with lowangle boundaries above approximately $0.05^{\circ}$ can be distinguished and are validated in the forward projection. An example of such is provided in Fig. 8, where combinations of diffractions spots of individual grains contributing to a cluster are clearly separable and distinguishable in various projections for different lattice planes, although they partially overlap.

\section{Discussion}

The presented reconstruction scheme is formulated in a general framework and is independent of the acquisition geometry and X-ray spectrum used. Though only the reconstruction for the LabDCT case with a polychromatic cone beam has been addressed, the reconstruction scheme could be adapted to classical synchrotron 3DXRD or DCT techniques, as these can be seen as special cases utilizing a parallel monochromatic beam. For a low-resolution spatial discreti-

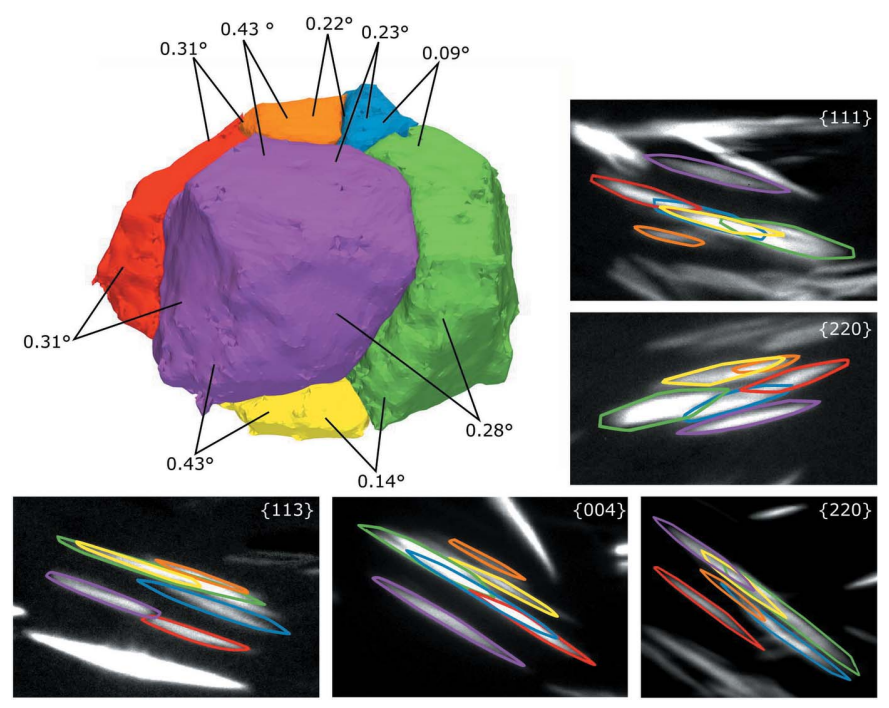

Figure 8

Grain cluster with low-angle grain boundaries and samples of their forward-projected convex hulls, coloured according to the grain. zation, the reconstruction scheme is able to produce a meaningful result within minutes, which allows one to fine-tune binarization and reconstruction parameters interactively and on the fly.

Compared with classical synchrotron techniques, similar known limitations on and requirements for samples apply in principle (King et al., 2013). Grain size, mosaicity or lattice deformation might pose greater limitations, as the polychromatic cone beam forms more complex diffraction contrast patterns and reveals more information captured simultaneously than a monochromatic beam. Owing to the lack of brilliance of a laboratory source compared with a synchrotron source, the minimum scattering volume of grains that is required to form diffraction spots with a suitably high signalto-noise ratio on the detector is assumed to be at least two or three times the spherical equivalent diameter of what the DCT technique at a synchrotron can resolve. Depending on the diffracted intensities of the material this corresponds to a minimum grain size of the order of $>20-40 \mu \mathrm{m}$ with the instrumentation currently used. The elongation of the diffraction spots on the detector due to the Laue-focusing effect allows for up to 400-500 grains to be illuminated simultaneously while maintaining an acceptable degree of spot overlap ( $c f$. McDonald et al., 2015). Although the current reconstruction model does not account for lattice imperfections across grains, they can be tolerated at the expense of the reliability and quality of the reconstructed grain map up to a certain point, where the diffraction spots formed on the detector are clearly extractable and separable. Lattice defects, deformations and mosaicity immediately affect pattern quality, but can be overcome by reducing the information content by the reducing illuminated volume. Exploiting the Laue-focusing geometry increases the angular sensitivity and allows subgrain boundaries $>0.05-0.1^{\circ}$ to be resolved depending on working distances, sample and grain sizes. Furthermore, the crystallography of the sample must be known in advance, which can pose a challenge for non-cubic systems.

Though the method optimizes only the completeness, complex grain shapes can be obtained as the grains assembling the microstructure are constrained by adjacent grains. Appropriate binarization of the diffraction contrast patterns is thus a critical and important step in order to avoid an over or under segmentation which jeopardizes completeness. The LabDCT reconstruction based on a simplified binary diffraction model reveals good agreement with and complements the well established absorption tomography reconstruction.

\section{Conclusion}

A fast and versatile reconstruction scheme is presented, extending the recently introduced LabDCT imaging modality on a laboratory X-ray microscope to capture the crystallographic orientation and morphology of a polycrystalline microstructure. The shape of the grains obtained from LabDCT is verified for correctness by means of an absorption contrast reconstruction. Large grain maps can be stitched 
together from several reconstructions. Absorption and phasecontrast reconstructions, which are the base imaging modalities on the same X-ray microscope, can be combined with the LabDCT reconstruction, enabling correlative analysis. The LabDCT implementation closes the gap to synchrotron grain mapping and can routinely be used in the laboratory.

\section{Acknowledgements}

The authors would like to thank Jules Dake, Ulm University, for providing the aluminium-copper sample. The authors also would like to acknowledge the comments of the reviewers which helped to improve the manuscript.

\section{References}

Aarle, W. van, Ludwig, W., King, A. \& Penumadu, D. (2015). J. Appl. Cryst. 48, 334-343.

Bachmann, F., Hielscher, R., Jupp, P. E., Pantleon, W., Schaeben, H. \& Wegert, E. (2010). J. Appl. Cryst. 43, 1338-1355.

Bachmann, F., Hielscher, R. \& Schaeben, H. (2011). Ultramicroscopy, 111, 1720-1733.

Bernier, J. V., Barton, N. R., Lienert, U. \& Miller, M. P. (2011). J. Strain Anal. Eng. Des. 46, 527-547.

Bleuet, P., Welcomme, E., Dooryhée, E., Susini, J., Hodeau, J. L. \& Walter, P. (2008). Nat. Mater. 7, 468-472.

Dake, J. M., Oddershede, J., Sørensen, H. O., Werz, T., Shatto, J. C., Uesugi, K., Schmidt, S. \& Krill, C. E. (2016). Proc. Natl Acad. Sci. USA, 113, E5998-E6006.

Ferreira Sanchez, D., Villanova, J., Laurencin, J., Micha, J.-S., Montani, A., Gergaud, P. \& Bleuet, P. (2015). J. Appl. Cryst. 48, 357-364.

Friedel, G. (1913). C. R. Acad. Sci. 157, 1533-1536.

Guinier, A. \& Tennevin, J. (1949). Acta Cryst. 2, 133-138.

Hayashi, Y., Hirose, Y. \& Seno, Y. (2015). J. Appl. Cryst. 48, 10941101.

Herbig, M., King, A., Reischig, P., Proudhon, H., Lauridsen, E. M., Marrow, J., Buffière, J. \& Ludwig, W. (2011). Acta Mater. 59, 590601.

Hofmann, F., Abbey, B., Connor, L., Baimpas, N., Song, X., Keegan, S. \& Korsunsky, A. M. (2012). Int. J. Mater. Res. 103, 192-199.

Hofmann, F., Song, X., Abbey, B., Jun, T.-S. \& Korsunsky, A. M. (2012). J. Synchrotron Rad. 19, 307-318.

Holzner, C., Lavery, L., Bale, H., Merkle, A., McDonald, S., Withers, P., Zhang, Y., Jensen, D. J., Kimura, M., Lyckegaard, A., Reischig, P. \& Lauridsen, E. M. (2016). Microsc. Today, 24, 34-43.

Ice, G. E., Larson, B. C., Yang, W., Budai, J. D., Tischler, J. Z., Pang, J. W. L., Barabash, R. I. \& Liu, W. (2005). J. Synchrotron Rad. 12, 155-162.

Johnson, G., King, A., Honnicke, M. G., Marrow, J. \& Ludwig, W. (2008). J. Appl. Cryst. 41, 310-318.

King, A., Herbig, M., Ludwig, W., Reischig, P., Lauridsen, E. M., Marrow, T. \& Buere, J. Y. (2010). Nucl. Instrum. Methods Phys. Res. $B, \mathbf{2 6 8}, 291-296$.
King, A., Johnson, G., Engelberg, D., Ludwig, W. \& Marrow, J. (2008). Science, 321, 382-385.

King, A., Reischig, P., Adrien, J. \& Ludwig, W. (2013). J. Appl. Cryst. 46, 1734-1740.

Larson, B. C., Yang, W., Ice, G. E., Budai, J. D. \& Tischler, J. Z. (2002). Nature, 415, 887-890.

Lauridsen, E. M., Schmidt, S., Suter, R. M. \& Poulsen, H. F. (2001). J. Appl. Cryst. 34, 744-750.

Li, S. F., Lind, J., Hefferan, C. M., Pokharel, R., Lienert, U., Rollett, A. D. \& Suter, R. M. (2012). J. Appl. Cryst. 45, 1098-1108.

Li, S. F. \& Suter, R. M. (2013). J. Appl. Cryst. 46, 512-524.

Lind, J. F. (2013). PhD thesis, Carnegie Mellon University, Pittsburgh, USA.

Ludwig, W., King, A., Reischig, P., Herbig, M., Lauridsen, E. M., Schmidt, S., Proudhon, H., Forest, S., Cloetens, P., du Roscoat, S. R., Buere, J. Y., Marrow, T. J. \& Poulsen, H. F. (2009). Mater. Sci. Eng. A, 524, 69-76.

Ludwig, W., Reischig, P., King, A., Herbig, M., Lauridsen, E. M., Johnson, G., Marrow, T. J. \& Buffière, J. Y. (2009). Rev. Sci. Instrum. 80, 033905 .

Ludwig, W., Schmidt, S., Lauridsen, E. M. \& Poulsen, H. F. (2008). J. Appl. Cryst. 41, 302-309.

McDonald, S., Reischig, P., Holzner, C., Lauridsen, E., Withers, P., Merkle, A. \& Feser, M. (2015). Sci. Rep. 5, 14665.

Moscicki, M., Kenesei, P., Wright, J., Pinto, H., Lippmann, T., Borbely, A. \& Pyzalla, A. R. (2009). Mater. Sci. Eng. A, 524, 64-68.

Poulsen, H. (2004). Three-Dimensional X-ray Diffraction Microscopy, Springer Tracts in Modern Physics, Vol. 205. Berlin, Heidelberg: Springer.

Poulsen, H. F. (2012). J. Appl. Cryst. 45, 1084-1097.

Poulsen, H. F. \& Fu, X. (2003). J. Appl. Cryst. 36, 1062-1068.

Poulsen, H. F., Garbe, S., Lorentzen, T., Juul Jensen, D., Poulsen, F. W., Andersen, N. H., Frello, T., Feidenhans'l, R. \& Graafsma, H. (1997). J. Synchrotron Rad. 4, 147-154.

Poulsen, H. F., Nielsen, S. F., Lauridsen, E. M., Schmidt, S., Suter, R. M., Lienert, U., Margulies, L., Lorentzen, T. \& Juul Jensen, D. (2001). J. Appl. Cryst. 34, 751-756.

Sanchez, D. F., Laloum, D., Weleguela, M. L. D., Ulrich, O., Audoit, G., Grenier, A., Micha, J., Robach, O., Lorut, F., Gergaud, P. \& Bleuet, P. (2014). J. Appl. Phys. 116, 163509.

Schmidt, S. (2014). J. Appl. Cryst. 47, 276-284.

Schmidt, S., Nielsen, S. F., Gundlach, C., Margulies, L., Huang, X. \& Jensen, D. J. (2004). Science, 305, 229-232.

Sharma, H., Huizenga, R. M. \& Offerman, S. E. (2012). J. Appl. Cryst. 45, 705-718.

Sørensen, H. O., Schmidt, S., Wright, J. P., Vaughan, G. B. M., Techert, S., Garman, E. F., Oddershede, J., Davaasambuu, J., Paithankar, K. S., Gundlach, C. \& Poulsen, H. F. (2012). Z. Kristallogr. 227, $63-$ 78.

Suter, R. M., Hennessy, D., Xiao, C. \& Lienert, U. (2006). Rev. Sci. Instrum. 77, 1-12.

Viganò, N., Ludwig, W. \& Batenburg, K. J. (2014). J. Appl. Cryst. 47, 1826-1840.

Viganò, N., Nervo, L., Valzania, L., Singh, G., Preuss, M., Batenburg, K. J. \& Ludwig, W. (2016). J. Appl. Cryst. 49, 544-555.

Wycko, R. W. G. (1963). Crystal Structures, 2nd ed. New York: Interscience Publishers. 